Case Report

\title{
Sepsis with cerebral lupus.... or is it? A case report on Cryptococcaemia
}

\author{
MN Jayawardena ${ }^{1}$, S Sigera $^{1}$, B Rathnayake ${ }^{2}$, RH Lewkebandara ${ }^{2}$, \\ L Rajakaruna $^{2}$, P Jayasekera ${ }^{1}$ \\ Sri Lankan Journal of Infectious Diseases 2018 Vol.8 (2):137-141
}

DOI: http://dx.doi.org/10.4038/sljid.v8i2.8214

\begin{abstract}
We present a patient with SLE, with multiple comorbidities, whose blood cultures yielded Cryptococcus neoformans. Blood cultures became positive only after twelve days of hospital admission. The patient died within two days of starting Amphotericin B. Cryptococci are an important cause of infection in SLE. Only about $10 \%$ to $30 \%$ of those with cryptococcal disease have the organism isolated by blood cultures. Due to its non-specific clinical presentation, cryptococcal infection in SLE can be misdiagnosed as psychosis due to steroids, cerebral lupus and infections due to other non-fungal pathogens. This may lead to inappropriate therapy and delays in administration of antifungal agents. Therefore, cryptococcal infection should be considered when SLE patients present with sepsis irrespective of the presence of features of meningism.
\end{abstract}

Keywords: Cryptococcus, Systemic Lupus Erythematosus, Fungal, Sepsis

\section{Introduction}

Patients with Systemic Lupus Erythematosus (SLE) are prone to infections and have a poorer prognosis when they have fungal infections. ${ }^{1}$ Among fungi, Candida species and Cryptococcus neoformans have been found to be the commonest. Importantly, cryptococcal meningitis is the foremost cause of mortality $(85.7 \%)$ among SLE patients due to invasive fungal infections. ${ }^{2}$

\footnotetext{
${ }^{1}$ Medical research Institute, Colombo 8, Sri Lanka

${ }^{2}$ District General Hospital, Badulla, Sri Lanka

Address for correspondence: Dr.Naamal Jayawardena, Medical research Institute, Colombo 8, Sri Lanka, Tel: +94077374599 Email: naamaljay@gmail.com (iD http://orcid.org/0000-0003-1928-8086

Received 25 April 2018 and revised version accepted 31 August 2018 


\section{Case history}

A 33-year-old female, a diagnosed patient with SLE and lupus nephritis for nearly 10 years, presented to the local hospital complaining of fever and generalized body swelling of three days duration. Her disease was in remission, and she was on maintenance doses of mycophenolate mofetil. She had been living in Middle East, where she had been supposedly treated for a cardiomyopathy and bone marrow suppression. Unfortunately, information was scarce regarding this episode.

On admission, she was conscious and rational, but had a rapidly progressive pneumonia, right lower limb cellulitis with features suggestive of sepsis.

Her initial full blood count was $8.1 \times 10^{3} / \mathrm{mm}^{3}$ with $85 \%$ neutrophils, haemoglobin $7.4 \mathrm{~g} / \mathrm{dL}$ and platelet count $90 \times 10^{3} / \mathrm{mm}^{3}$. On admission her C-reactive protein was elevated to $114 \mathrm{mg} / \mathrm{L}$, while the urine full report had 3+ albumin, 6-8 pus cells / hpf and 5 red cells / hpf. She was also found to have acute respiratory distress syndrome (ARDS) and pulmonary embolism. She had a low urine output, with a serum creatinine of $221 \mu \mathrm{mol} / \mathrm{L}(60-104 \mu \mathrm{mol} / \mathrm{L})$. Her initial blood culture revealed a mixed growth of Acinetobacter species and coliforms of which the former was resistant to all antibiotics tested, including carbapenems. Colistin was not tested due to unavailability of Estrips or paper discs. The coliforms were sensitive to carbapenems. The endotracheal secretions failed to reveal a pathogen.

The patient was admitted to the intensive care unit (ICU) for further management. A central venous line was inserted for monitoring, and she was started on IV meropenem $1 \mathrm{~g} 8$ hourly, oral mycophenolate mofetil 500mg twice daily, along with pulsed methyl prednisolone and IV cyclophosphamide $600 \mathrm{mg}$ pulses.

Despite the above, the patient's CRP levels remained high $(127 \mathrm{mg} / \mathrm{L})$. Her neutrophil leukocytosis was also increasing, while high resolution CT scan of the chest revealed an organizing but ongoing pneumonia.

On the $12^{\text {th }}$ day after admission, her blood culture (BacT/ALERT ${ }^{\circledR}$ bioMérieux, U.S.A) became positive after 24 hours of incubation for Gram positive, oval shaped yeast cells with budding. The same organism was isolated in the repeat blood culture taken the next day. The isolated organism was suspected to be capsulated, hence India ink staining was performed (Fig 1a). The blood culture bottle was then dispatched to the reference laboratory as the required technical skills and experience in interpretation of the stain was not available locally. 


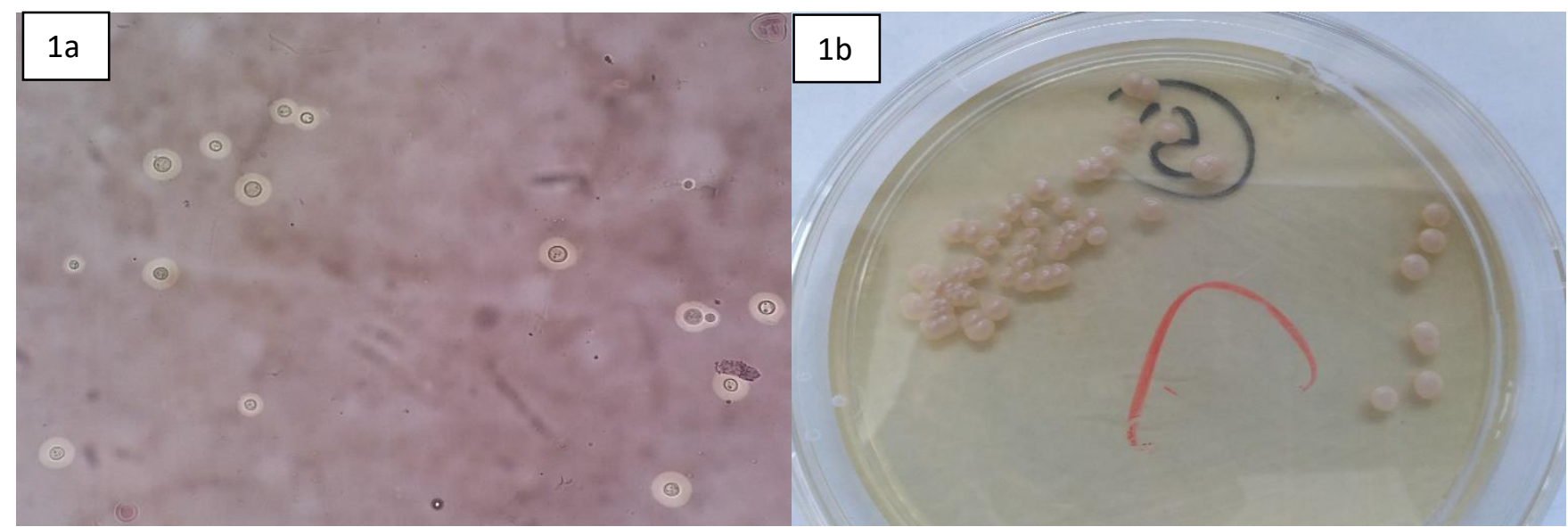

Figure 1 (a) The direct smear of the isolate in India ink b) culture in Sabouraud Dextrose agar after overnight incubation (taken at the Dept. of Mycology, MRI)

The patient was initially started on IV fluconazole 400mg daily but was changed to IV liposomal amphotericin B $3 \mathrm{mg} / \mathrm{kg}$ daily after a presumptive diagnosis of cryptococcal infection was made. A lumbar puncture was not performed due to her unstable condition.

The isolates were sent to the Mycology Reference Laboratory at the Medical Research Institute for further identification. Both isolates were identified as Cryptococcus neoformans based on culture morphology, demonstration of a capsule, positive urease test, carbohydrate assimilation and API Candida® (Biomerieux) results.

The patient meanwhile developed pulmonary haemorrhages 17 days after admission. Two days afterwards, therapeutic plasma exchange was performed, but unfortunately, she died the same day.

The timeline of the patient's clinical course is given below

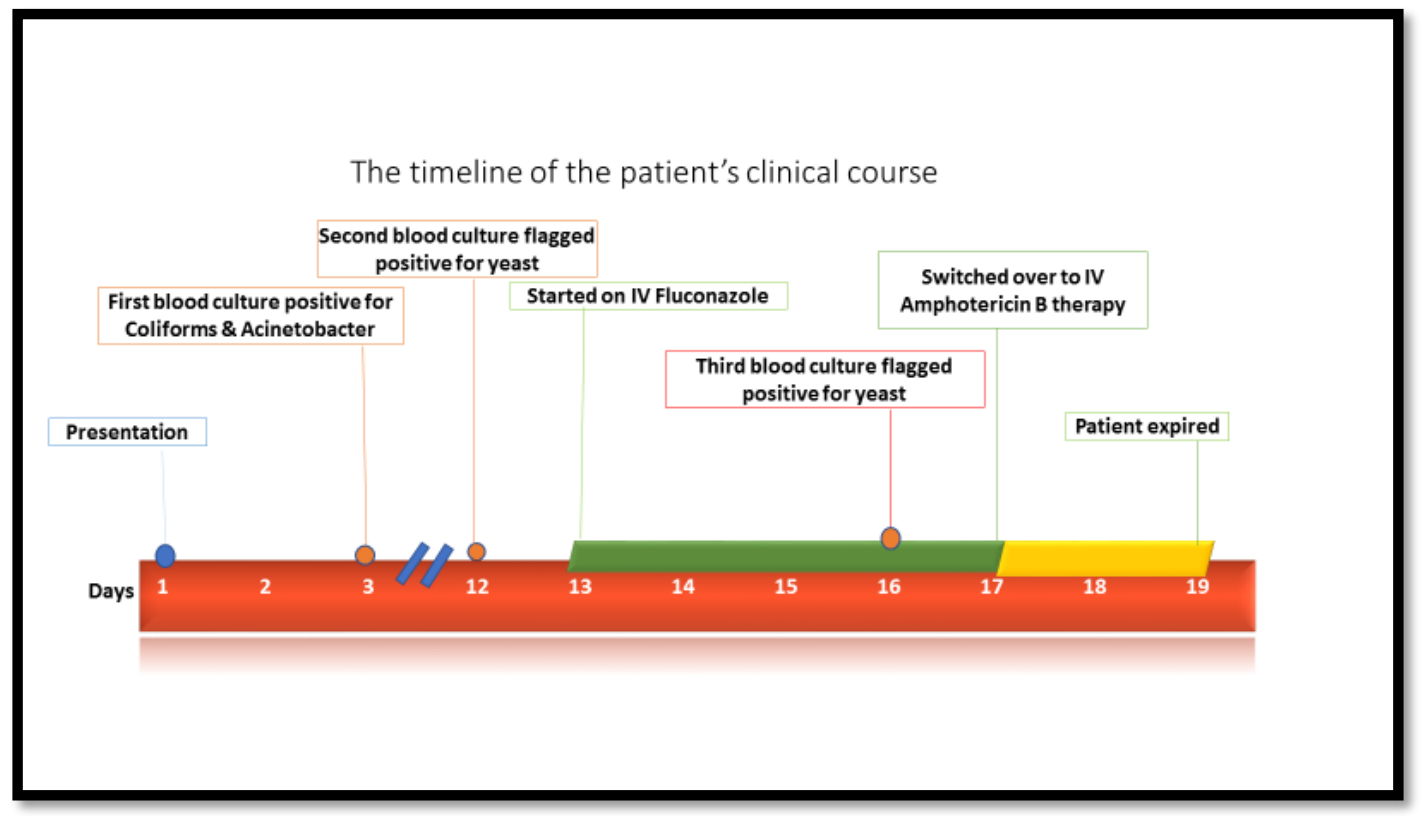




\section{Discussion}

SLE has the probability of affecting all organs in the body, causing much morbidity and mortality. Even though survival has improved in recent years due to early diagnosis and better treatment options, patients still have a three-fold increase in all-cause premature mortality, compared with the general population. Among the causes with highest mortality, is renal disease and infection, causing eight and five times increased mortality respectively, compared with the general population. ${ }^{1}$

Both disease activity and therapy in SLE increases the patient's risk of infection, as steroids and immunosuppressive therapy are used increasingly in those with severe and active disease. It has been found that treatment with steroids and immunosuppressive drugs are risk factors for infection in SLE patients. ${ }^{3}$ These patients are prone to infections by Gram positive cocci, Gram negative bacilli, Candida albicans, Cryptococcus neoformans, Pneumocystis jirovecii and Mycobacterium tuberculosis among others. It has also been noted that these patients have a poorer prognosis when they have fungal infections. Among fungal pathogens, Candida species and $C$. neoformans have been found to be the commonest. ${ }^{4}$

Cryptococcal meningitis is the foremost cause of mortality $(85.7 \%)$ in patients with SLE who have invasive fungal infections. ${ }^{5}$ The reasons for this are multifactorial and include its non-specific clinical presentation in the early stage of infection, misjudging the risk for cryptococcal meningitis and misdiagnosis as psychosis due to steroids, cerebral lupus and other non-fungal pathogens. ${ }^{2}$ This leads to inappropriate therapy and delays in administration of antifungal agents.

Cryptococcal meningitis should be a consideration when patients with SLE present with headache, nausea, vomiting or fever. Despite antifungal therapy, the consequences of cryptococcal meningitis are disastrous, as mortality is around 40-50\%, even with antifungal therapy. ${ }^{5}$

C. neoformans can be isolated from the blood, as in our patient, only in around $10 \%$ to $30 \%$ patients with cryptococcal disease. ${ }^{6}$ It should be noted that when this happens, in those with cryptococcal meningitis, it heralds a poor prognosis. Also, cryptococcaemia can induce a systemic inflammatory response, and sepsis is its most common manifestation. Therefore, early diagnosis and initiation of antifungal treatment promptly is essential. We presume our patient had concomitant cryptococcal meningitis, as around $82 \%$ of those with cryptococcaemia have been found to have same. ${ }^{6}$ Hence, the treatment regime for cryptococcaemia is essentially the same as for cryptococcal meningitis. ${ }^{7}$

According to guidelines published by the Infectious Disease Society of America (IDSA), treatment induction is with IV Amphotericin B deoxycholate combined with oral flucytosine, (or fluconazole in low resource settings) where lipid formulations of Amphotericin B could be substituted in the second 2 weeks. This is followed by a consolidation phase, where oral fluconazole is prescribed for 8 weeks and maintenance therapy where oral fluconazole is given for 6-12 months.

This case highlights an instance where cryptococcal disease was diagnosed via blood cultures. Although in disease remission, our patient with SLE was at a higher risk for infectious complications, owing to being immunosuppressed. Although it was possible that she had central 
nervous system infection, this could not be proven. Sepsis in this patient may have been due to the bacterial infection, or cryptococcaemia or both.

Many issues confronted the optimal management of this patient. Rapid diagnosis of cryptococcal infection using the cryptococcal antigen test was not possible at the local hospital. Sending samples to the reference laboratory situated nearly $230 \mathrm{~km}$ away at short notice was difficult. Lack of local expertise in identification of cryptococci, in particular, interpretation of the India ink stain was also problematic. Recommended treatment strategies could not be implemented immediately as obtaining the necessary antifungal drugs was difficult and precious time was lost in the process.

In conclusion, we present a patient with SLE, with multiple comorbidities, where cryptococcal infection was diagnosed via blood cultures. She eventually died due to the disease process, with infection no doubt playing a major role. This case highlights the importance of including cryptococcal infection in the differential diagnosis of sepsis in immunosuppressed patients.

\section{References}

1. Yurkovich M, Vostretsova K, Chen W, et al. Overall and cause-specific mortality in patients with systemic lupus erythematosus: A meta-analysis of observational studies. Arthritis Care Res 2014; 66(4):608-616. doi: http://dx.doi.org/10.1002/acr.22173.

2. Fang W, Chen M, Liu J, et al. Cryptococcal meningitis in systemic lupus erythematosus patients: pooled analysis and systematic review. Emerg Microbes Infect (2016) 5, e95;

doi: http://dx.doi.org/ 10.1038/emi.2016.93

3. Gladman DD, Hussain F, Ibanez D, et al. The nature and outcome of infection in systemic lupus erythematosus. Lupus 2002; 11:234 - 239. doi: http://dx.doi.org/10.1191/0961203302lu170oa

4. Chen HS, Tsai WP, Leu HS, et al. Invasive fungal infection in systemic lupus erythematosus: an analysis of 15 cases and a literature review. Rheumatology 2007; 46:539-544 doi: http://dx.doi.org/10.1093/rheumatology/kel343

5. Matsumura M, Kawamura R, Inoue R, et al. Case report: Concurrent presentation of cryptococcal meningoencephalitis and systemic lupus erythematosus. Mod Rheumatol 2011; 21:305-308 doi: http://dx.doi.org/10.1007/s10165-010-0383-6.

6. Jean SS, Fang CT, Shau WY, et al. Cryptococcaemia: clinical features and prognostic factors. $Q J$ Med 2002; 95:511-518 doi: http://dx.doi.org/10.1093/qjmed/95.8.511

7. Perfect JR, Dismukes WE, Dromer F, et al. Clinical practice guidelines for the management of cryptococcal disease, Clinical Infectious Diseases 2010; 50:291-322.

doi: http://dx.doi.org/10.1086/649858 